\title{
Interaction of the Cytosolic Domains of sorLA/LR11 with the Amyloid Precursor Protein (APP) and $\beta$-Secretase $\beta$-Site APP-Cleaving Enzyme
}

\author{
Robert Spoelgen, ${ }^{1}$ Christine A. F. von Arnim, ${ }^{1}$ Anne V. Thomas, ${ }^{1}$ Ithan D. Peltan, ${ }^{1}$ Mirjam Koker, ${ }^{1}$ Amy Deng, ${ }^{1}$ \\ Michael C. Irizarry, ${ }^{1}$ Olav M. Andersen, ${ }^{2}$ Thomas E. Willnow, ${ }^{2}$ and Bradley T. Hyman ${ }^{1}$ \\ ${ }^{1}$ Alzheimer's Disease Research Laboratory, Massachusetts General Hospital, Harvard Medical School, Charlestown, Massachusetts 02129, and 2Max \\ Delbrück Center for Molecular Medicine, D-13125 Berlin, Germany
}

sorLA is a recently identified neuronal receptor for amyloid precursor protein (APP) that is known to interact with APP and affect its intracellular transport and processing. Decreased levels of sorLA in the brain of Alzheimer's disease (AD) patients and elevated levels of amyloid- $\beta$ peptide $(\mathrm{A} \beta)$ in sorLA-deficient mice point to the importance of the receptor in this neurodegenerative disorder. We analyzed APP cleavage in an APP-shedding assay and found that both sorLA and, surprisingly, a sorLA tail construct inhibited APP cleavage in a $\beta$-site APP-cleaving enzyme (BACE)-dependent manner. In line with this finding, sorLA and the sorLA tail significantly reduced secreted $A \beta$ levels when BACE was overexpressed, suggesting that sorLA influences $\beta$-cleavage. To understand the effect of sorLA on APP cleavage by BACE, we analyzed whether sorLA interacts with APP and/or BACE. Because both full-length sorLA and sorLA C-terminal tail constructs were functionally relevant for APP processing, we analyzed sorLA-APP for a potential cytoplasmatic interaction domain. sorLA and C99 coimmunoprecipitated, pointing toward the existence of a new cytoplasmatic interaction site between sorLA and APP. Moreover, sorLA and BACE also coimmunoprecipitate. Thus, sorLA interacts both with BACE and APP and might therefore directly affect BACE-APP complex formation. To test whether sorLA impacts BACE-APP interactions, we used a fluorescence resonance energy transfer assay to evaluate BACE-APP interactions in cells. We discovered that sorLA significantly reduced BACE-APP interactions in Golgi. We postulate that sorLA acts as a trafficking receptor that prevents BACE-APP interactions and hence BACE cleavage of APP.

Key words: sorLA; Alzheimer's disease; amyloid- $\beta$; BACE; amyloid precursor protein; LR11

\section{Introduction}

Alzheimer's disease $(\mathrm{AD})$ is a neurodegenerative disorder with neurofibrillary tangles and amyloid plaques as its pathological hallmarks (Selkoe, 2001). Amyloid plaques are mainly composed of aggregated amyloid- $\beta$ peptide $(\mathrm{A} \beta)$, which is derived from the amyloid precursor protein (APP) by sequential proteolysis by $\beta$-secretase $\beta$-site of APP-cleaving enzyme (BACE) and presenilin 1 (PS1) (Hussain et al., 1999; Sinha et al., 1999; Vassar et al., 1999; Wolfe et al., 1999; Yan et al., 1999; Lin et al., 2000; Xia et al., 2000; Wolfe and Kopan, 2004). In recent studies, sorLA/LR11, a highly conserved type I membrane receptor, has been identified that impacts APP processing (Andersen et al., 2005). Overexpression of sorLA in neurons shifts the subcellular localization of APP to the Golgi apparatus and reduces the production of secreted $\mathrm{A} \beta$. The pathogenic relevance of sorLA in Alzheimer's disease is demonstrated by elevated levels of $A \beta$ in sorLA-deficient mice

Received Sept. 13, 2005; revised Nov. 9, 2005; accepted Nov. 14, 2005.

This work was supported by National Institutes of Health Grant AG 12406, Deutsche Forschungsgemeinschaft Research Fellowships AR379/1-1 (to C.A.F.v.A.) and TH 1129/1-1 (to A.V.T.), and the J. D. French Alzheimer's Foundation (to M.C.I.). We thank S. F. Lichtenthaler for the SEAP-APP construct.

Correspondence should be addressed to Dr. Bradley T. Hyman, Department of Neurology/Alzheimer Unit, 114 16th Street, Charlestown, MA 02129. E-mail: bhyman@partners.org.

DOI:10.1523/JNEUROSCI.3882-05.2006

Copyright $\odot 2006$ Society for Neuroscience $\quad$ 0270-6474/06/260418-11\$15.00/0
(Andersen et al., 2005) and by the reduced expression of sorLA in the brain of $\mathrm{AD}$ patients (Scherzer et al., 2004; Andersen et al., 2005).

sorLA is a member of a novel family of vacuolar protein sorting 10 protein $(\mathrm{Vps} 10 \mathrm{p})$ receptors, which contain a domain with high homology to the yeast-sorting protein Vps10p (Yamazaki et al., 1996; Jacobsen et al., 2001). The 54-residue cytoplasmatic domain is highly similar to that of the cation-independent mannose 6-phosphate receptor and is also capable of binding Golgi apparatus-localized $\gamma$-ear-containing ARF-binding (GGA) proteins (Jacobsen et al., 2002). Because the Vps10p domain and the interaction of GGA adaptor proteins with the mannose 6-phosphate receptor have been shown to mediate intracellular protein trafficking (Puertollano et al., 2001; Takatsu et al., 2001; Zhu et al., 2001), it is plausible that sorLA is a sorting receptor involved in APP trafficking and processing in neurons. In line with these structural features of sorLA, the receptor mainly affects trafficking and processing rather than the synthesis of APP (Andersen et al., 2005). Overexpression of sorLA in neuronal SY5Y cells leads to a decrease in cell surface APP and coincides with significantly lower production of $\operatorname{sAPP} \alpha, \operatorname{sAPP} \beta$, and $\mathrm{A} \beta$ compared with wild-type cells. Overexpression of a mutant form of the receptor lacking the sorLA cytoplasmatic domain not only points to a role of sorLA in APP trafficking but also demonstrates 
that the lumenal N-terminal parts of sorLA and APP interact; this was confirmed by surface plasmon resonance analysis (Andersen et al., 2005).

sorLA could act as an endocytic receptor for APP, enhancing APP turnover or directing APP to non-A $\beta$-generating degradation pathways. Alternatively, sorLA could also impact APP trafficking or access to BACE or $\gamma$-secretase, precluding $\mathrm{A} \beta$ generation. In the present study, we observe a second site of interaction between APP and sorLA involving the C-terminal domains of APP and sorLA. Moreover, we show that the sorLA tail alone is sufficient to influence APP processing and provide additional evidence that sorLA influences the production of sAPP $\beta$ and $A \beta$. Surprisingly, we found that sorLA also interacts with BACE. Our results suggest that sorLA acts as a crucial regulatory molecule for BACE-APP interactions.

\section{Materials and Methods}

Generation of expression constructs. The generation of sorLA (human cDNA in pcDNA3.1), C-terminal myc-tagged APP695 (APP-myc), C99 (C99-myc), secreted alkaline phosphatase (SEAP) fused to the $\mathrm{N}$ terminus of APP695 (SEAP-APP) and C-terminal V5-tagged BACE (BACEV5) constructs was performed as described previously (Kinoshita et al., 2001; Lichtenthaler et al., 2003; von Arnim et al., 2004; Andersen et al., 2005). The sorLA tail construct that encodes bp 6355-6442, according to GenBank accession number NM_003105, was amplified and ligated into expression vector pSecTag2B (Invitrogen, Carlsbad, CA) by PCR using the following primers: $5^{\prime}$-CCGCTCGAGTCTGGGGTCTGGTGCAGATGCA-3' and 5'-CGTCTAGAGGCTATCACCATGGGGACGTC-3'.

The PCR product was inserted into the $\mathrm{XhoI} / \mathrm{XbaI}$ restriction sites of modified pSecTag.

To generate the C-terminal green fluorescent protein (GFP)-tagged or flag-tagged construct for sorLA, the sorLA pcDNA3.1 construct was modified using the QuickChange site-directed mutagenesis kit (Stratagene, La Jolla, CA) to insert an AgeI restriction site. GFP was amplified by PCR and inserted into the new C-terminal AgeI site using the primer pair: 5'-CTAACCGGTATGGTGAGCAAGGGCGAGG-3' and 5'-CTAACCGGTTTACTTGTACAGCTCGTCCATGC-3'.

C-terminal flag-tagged sorLA was generated with a linker sequence using the primer pair: 5'-CCGGTGATTACAAGGATGACGACGATAAGTGAA- $3^{\prime}$ and $5^{\prime}$-CCGGTTCACTTATCGTCGTCATCCTTGTAATCA-3'.

The GYENPTY site mutant of C99 was generated by substitution of the tyrosine residues 86 and 91 (of C99) to alanine of C99-myc using the QuickChange site-directed mutagenesis kit (Stratagene). Authenticity of all PCR products and the PCR-generated constructs was confirmed by DNA sequencing.

Cell culture conditions and transient transfection. For this study, we used Chinese hamster ovary (CHO) 7W (Wolfe et al., 1999), mouse neuroblastoma N2a cells and human embryonic kidney 293 (HEK293) cells. CHO 7W and N2a cells were grown in OptiMEM (Invitrogen) with 5\% FBS and HEK293 cells were grown in DMEM with 10\% FBS in an incubator at $37 \mathrm{C}$ and $5 \% \mathrm{CO}_{2}$. Transient transfection of the cells was performed using a lipid-based method (FuGENE 6; Roche Applied Science, Indianapolis, IN) according to the instructions of the manufacturer.

Primary neuronal cultures were generated from CD1 mice at embryonic day 15-16, as described previously (Berezovska et al., 2003). Hippocampal and cortical neurons were dispersed in neurobasal media (Invitrogen) containing 10\% FBS and plated onto poly-L-lysine coated four-well glass slides. The media was exchanged after $1 \mathrm{~h}$ to neurobasal media containing $1 \times \mathrm{B} 27$, in which the cells were cultured for $10-12 \mathrm{~d}$ in vitro.

Immunocytochemistry. Immunostaining was performed $24 \mathrm{~h}$ after transient transfection. Cells were fixed in $4 \%$ paraformaldehyde for 10 min, washed in Tris-buffered saline (TBS), pH 7.3, permeabilized with $0.5 \%$ Triton X-100 for $20 \mathrm{~min}$, and blocked with $1.5 \%$ normal goat serum for $1 \mathrm{~h}$. To detect the localization of APP695-myc or C99-myc and sorLA, transfected cells were immunostained using monoclonal antibody 8E5 (raised against 444-592 residues of APP770; a gift from Elan Pharmaceuticals, San Francisco, CA) and rabbit N-terminal anti-SOL-sorLA antibody (1:2000) or N-terminal rab LA sorLA antibody (provided by Anders Nykjaer, University of Aarhus, Aarhus, Denmark) or monoclonal 13G8 antibody (raised against 676-695 residues of APP770; 1:500; gift from Elan Pharmaceuticals), monoclonal 9E10 anti-c-myc antibody (Abcam, Cambridge, UK) and rab C-terminal anti-sorLA (raised against 20 C-terminal residues of sorLA; provided by Claus Munck Petersen, University of Aarhus, Aarhus, Denmark). To detect the subcellular localization of BACE, cells transfected with BACE-V5 were immunostained with anti-V5 monoclonal antibody (Sigma, St. Louis, MO). Endogenous BACE was stained or immunporecipitated by rabbit anti-BACE $\mathrm{N}$-terminal antibody (Calbiochem, La Jolla, CA). After three washes in TBS, Alexa 488- and cyanine 3 (Cy3)-conjugated secondary antibodies [from Invitrogen and Jackson ImmunoResearch (West Grove, PA)] were applied for $1 \mathrm{~h}$ at room temperature. Immunostained cells were coverslipped using GVA (glycerol vinyl alcohol) mounting solution (Zymed, South San Francisco, CA) before fluorescence lifetime imaging microscopy (FLIM) measurements or confocal imaging with appropriate filters using a Bio-Rad (Hercules, CA) 1024 confocal microscope.

Fluorescence resonance energy transfer measurements using FLIM. We applied a validated FLIM technique to quantitate protein-protein interactions using multiphoton microscopy (Bacskai et al., 2003; Berezovska et al., 2003). Images were acquired using a Bio-Rad Radiance 2000 multiphoton microscope. A mode-locked Ti:sapphire laser (Spectra Physics, San Jose, CA) sends a 100 fs pulse every 12 ns to excite the fluorophore. We used a high-speed Hamamatsu (Shizuoka, Japan) MCP5900 detector and hardware/software from Becker and Hickl (Berlin, Germany) to measure fluorescence lifetimes on a pixel-by-pixel basis. Excitation at $800 \mathrm{~nm}$ was empirically determined to excite Alexa 488 but not Cy3. Donor fluorophore (A488) lifetimes were fit to two exponential decay curves to calculate the fraction of fluorophores within each pixel that interacts with an acceptor. As a negative control, Alexa 488 lifetime was measured in the absence of acceptor (Cy3), which was equivalent to Alexa 488 alone or in solution. Statistical analysis of FLIM data were performed by Student's $t$ test after determination of normal distribution and variance.

Western blotting. N2a or HEK cells were transfected with APP695-myc, either empty vector or BACE-V5 and sorLA. For cell lysis, cells were treated with $1 \%$ Triton X-100 using Complete Protease Inhibitor (Roche Applied Science) and then loaded onto 4-20\% Tris-glycine polyacrylamide gels for separation under reducing and denaturating conditions. After gel separation, proteins were transferred to polyvinylidene difluoride (PVDF) membranes and labeled using IR700 and IR800 secondary antibodies (Rockland, Gilbertsville, PA). Proteins were visualized on a LI-COR (Lincoln, NE) Odyssey infrared detection system according to the instructions of the manufacturer.

Immunoprecipitation. Immunoprecipitation of either myc-tagged C99 or GFP-tagged sorLA were performed with $\mu$ MACS epitope isolation kit (Miltenyi Biotec, Auburn, CA) as recommended by the manufacturers. The magnetic beads were precoupled to either anti-myc or anti-GFP antibodies and were incubated with lysates for $30 \mathrm{~min}$ on ice. The complex consisting of beads and proteins was captured on a flow-through magnetic column and washed five times with cold lysis buffer. The proteins then were eluted with hot elution buffer. For negative controls, lysates from cells without the myc- or GFP-tagged protein were used. Lysates and immunoprecipitates were loaded onto 4-20\% Tris-glycine polyacrylamide gels (Novex, San Diego, CA) under denaturating and reducing conditions. The proteins were transferred to PVDF membranes (Millipore, Bedford, MA) and blocked in 5\% nonfat dried milk. sorLA was detected by rabbit anti-SOL-sorLA antibody, and C99 was detected by mouse 6E10 antibody (Signet, Dedham, MA). Secondary antibodies conjugated to infrared dyes were applied and detected using the LI-COR system.

Immunoprecipitation of BACE-V5 and sorLA were performed with BioMag goat anti-mouse or goat anti-rabbit IgG magnetic beads (PerSeptive Biosystems, Framingham, MA). N2a cells were cotransfected with BACE-V5 and sorLA and lysed in 1\% Triton X-100 buffer. Cell 
lysates were incubated with anti-V5 or anti-SOL-sorLA antibody-loaded beads for $3 \mathrm{~h}$ at $4^{\circ} \mathrm{C}$. For negative controls, beads were incubated with lysate or antibody alone. After the incubation, beads were washed and boiled in $2 \times$ Tris-glycine SDS sample buffer (Invitrogen). Supernatants and immunoprecipitates were loaded onto $4-20 \%$ Tris-glycine polyacrylamide gels (Novex) under denaturing conditions. Western blots were performed as described above using anti-V5 and rabbit SOL-sorLA antibody for detection.

Immunoprecipitation of endogenous sorLA by BACE was done with Sepharose beads. Beads and mouse brain lysates were incubated with anti-BACE $\mathrm{Ab}$ or PBS at $4^{\circ} \mathrm{C}$. After the supernatants were collected, beads were washed with $1 \%$ CHAPSO lysis buffer, boiled with $2 \times$ Tris-glycine SDS sample buffer and loaded onto $4-20 \%$ Tris-glycine polyacrylamide gels (Novex) under denaturing and reducing conditions. The proteins were transferred to PVDF membranes (Millipore), and sorLA was detected by rabbit SOL-sorLA antibody or C-terminal anti-sorLA antibody. Secondary antibodies conjugated to horseradish peroxidase were applied and visualized by chemiluminescence.

Internalizaion assay. CHO 7W (APP751 stable overexpressing cells, provided by D. Selkoe, Brigham and Women's Hospital, Boston, MA) were cultured on six-well plates and transfected with sorLA or empty vector pcDNA3.1. On the next day, cells were washed with cold PBS containing $1 \mathrm{mM} \mathrm{CaCl}_{2}$ and $1 \mathrm{~mm} \mathrm{MgCl}_{2}, 0.2 \% \mathrm{BSA}$, and $5 \mathrm{~mm}$ glucose and incubated for $30 \mathrm{~min}$ on ice with biotinylated 6E10 (Signet). Cells were then incubated at $37^{\circ} \mathrm{C}$ for different times to allow for endocytosis of 6E10. After endocytosis, surface biotin was masked using streptavidin (Roche Applied Science) for $1 \mathrm{~h}$ on ice and quenched with $0.5 \mathrm{mg} / \mathrm{ml}$ biocytin (Sigma). Cells were harvested in blocking buffer containing $1 \%$ Triton X-100, 0.1\% SDS, 0.2\% BSA, $50 \mathrm{~mm} \mathrm{NaCl}, 1$ mm Tris, $\mathrm{pH}$ 7.4, and incubated on IgG-coated 96-well plates at $4^{\circ} \mathrm{C}$ overnight. Plates were washed with PBS and streptavidin-peroxidase (Roche Applied Science) was applied for $1 \mathrm{~h}$ in blocking buffer. Finally, plates were washed and then incubated with $10 \mathrm{mg}$ of $o$-phenyldiamine $\mathrm{HCl}$ (Sigma), $10 \mu \mathrm{l}$ of $30 \% \mathrm{H}_{2} \mathrm{O}_{2}$ in $25 \mathrm{ml}$ of $50 \mathrm{~mm} \mathrm{Na}_{2} \mathrm{HPO}_{4}, 27 \mathrm{~mm}$ citrate, $\mathrm{pH}$ 5.0. The readout was done at $490 \mathrm{~nm}$ to determine internalized APP against surface APP.

APP ectodomain shedding assay. HEK293 cells cultured in 12-well plates were transfected in triplicate with a $\beta$-galactosidase $(\beta$-gal) reporter, SEAP-APP and either BACE, sorLA, sorLA tail, or combinations of either sorLA or sorLA tail with BACE. On the next day, media were changed and collected after $24 \mathrm{~h}$. SEAP activity in conditioned media was determined by a chemiluminesscent assay (Roche Applied Science) and normalized to $\beta$-gal. The SEAP assay was done to according to the instructions of the manufacturer, and $\beta$-gal activity was measured by hydrolysis of $o$-nitrophenylk- $\beta \mathrm{D}$-galactopyranoside in cells lysed with reporter lysis buffer (Promega, Madison, WI). To confirm consistent expression of SEAP-APP, BACE, and sorLA constructs, Western blotting was performed as described above.

$A \beta$ quantification. CHO 7W cells were transfected either with BACE, sorLA, sorLA tail or a combination of BACE and sorLA constructs. For $\mathrm{A} \beta$ quantification in the media, media was exchanged after $24 \mathrm{~h}$ and collected after another $24 \mathrm{~h}$. A $\beta 40$ levels were determined by sandwich ELISA using BNT77 (anti-A $\beta$ 11-28) as the capture antibody and horseradish peroxidase-linked BA27 as detection antibodies (Takeda Chemical Company, Osaka, Japan) (Fukumoto et al., 1999; Irizarry et al., 2004). Purified A $\beta 40$ (Bachem, King of Prussia, PA) was used as standard. The sensitivity of this ELISA assay is $1 \mathrm{pm} \mathrm{A} \beta$, and it is not affected by serum and plasma proteins because of the high affinity of the antibodies (Scheuner et al., 1996; Kosaka et al., 1997; Fukumoto et al., 2003). Cells were checked for consistent expression of constructs using Western blotting as described above.

\section{Results}

\section{Endocytosis of APP by sorLA}

In our previous study, we analyzed several aspects of the role of the putative sorting receptor sorLA in APP metabolism. We showed that overexpression of sorLA coincides with sequestration of APP in the Golgi and reduced cell surface exposure of APP

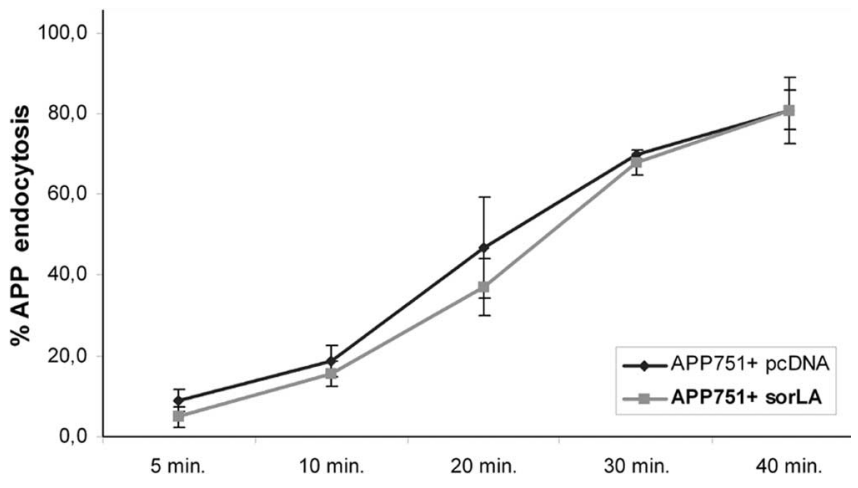

Figure 1. APP internalization assay shows that APP internalization is not dependent on sorLA. 7W CHO cells were transfected with either empty vector or sorLA. The uptake of cellsurface-biotinylated APP was assessed by determining internalized versus cell surface APP. The internalization of APP is indicated in percentage of cell surface APP over 40 min and revealed no sorLA-dependent uptake of APP. The results are representative of data of three independent assays. The means and SD of one assay performed in triplicate are shown.

(Andersen et al., 2005). Conceivably, sequestration may be achieved by sorLA-mediated retention of APP en route through the secretory pathway to the plasma membrane or by accelerated internalization of APP from the cell surface and recycling back to the Golgi. To investigate whether sorLA may act as an endocytic receptor mediating the internalization of cell surface APP, we assayed the uptake of biotinylated cell surface APP over time (Fig. $1)$. In these experiments, we did not detect a difference between sorLA-expressing and sorLA-deficient cells, because we observed a relative uptake of APP $\leq 80 \%$ in both conditions. These data indicate that sorLA activity does not influence APP uptake from the cell surface but rather affects the kinetics of APP trafficking through the Golgi.

\section{APP shedding assay}

Recently, we demonstrated that the sorLA overexpression causes a significant reduction of $\operatorname{sAPP} \alpha, \operatorname{sAPP} \beta$, and $\mathrm{A} \beta$ levels (Andersen et al., 2005) and that the extracellular domain of sorLA binds directly to APP. To investigate the molecular mechanisms that are responsible for reduced APP processing, we established an APP shedding assay. Based on this assay, we were able to analyze whether sorLA impacts BACE dependent APP cleavage. Additionally, we asked whether the sorLA C-terminal tail alone was sufficient to reduce APP cleavage. Therefore, we assessed APP cleavage by transfecting HEK293 cells with an APP construct, where a secreted alkaline phosphatase is fused to the N-terminal end of APP695 (SEAP-APP). Whenever this construct gets cleaved, the N-terminal fragment of APP is secreted into the media, and the amount of alkaline phosphatase activity in the media provides a sensitive indicator for sAPP production (Lichtenthaler et al., 2003). HEK293 cells were transfected with SEAP-APP and empty vector, BACE, sorLA-FLAG, sorLA-tailFLAG or both BACE and sorLA or sorLA-tail-FLAG. To normalize for transfection efficiency, a $\beta$-gal reporter construct was cotransfected, and the resulting SEAP activity in the media was normalized to $\beta$-gal activity. The SEAP activity for cells overexpressing sorLA or sorLA tail only was unchanged in comparison to the empty vector controls (Fig. $2 A$, lanes 1-3). However, when BACE was overexpressed as well, sorLA strongly reduced SEAPAPP cleavage by $50 \%$ in comparison to cells only transfected with BACE (Fig. 2A, lane 5). This is consistent with our previous findings (Andersen et al., 2005), in which sorLA inhibits sAPP production particular in SH-SY5Y cells, a cell line with high en- 


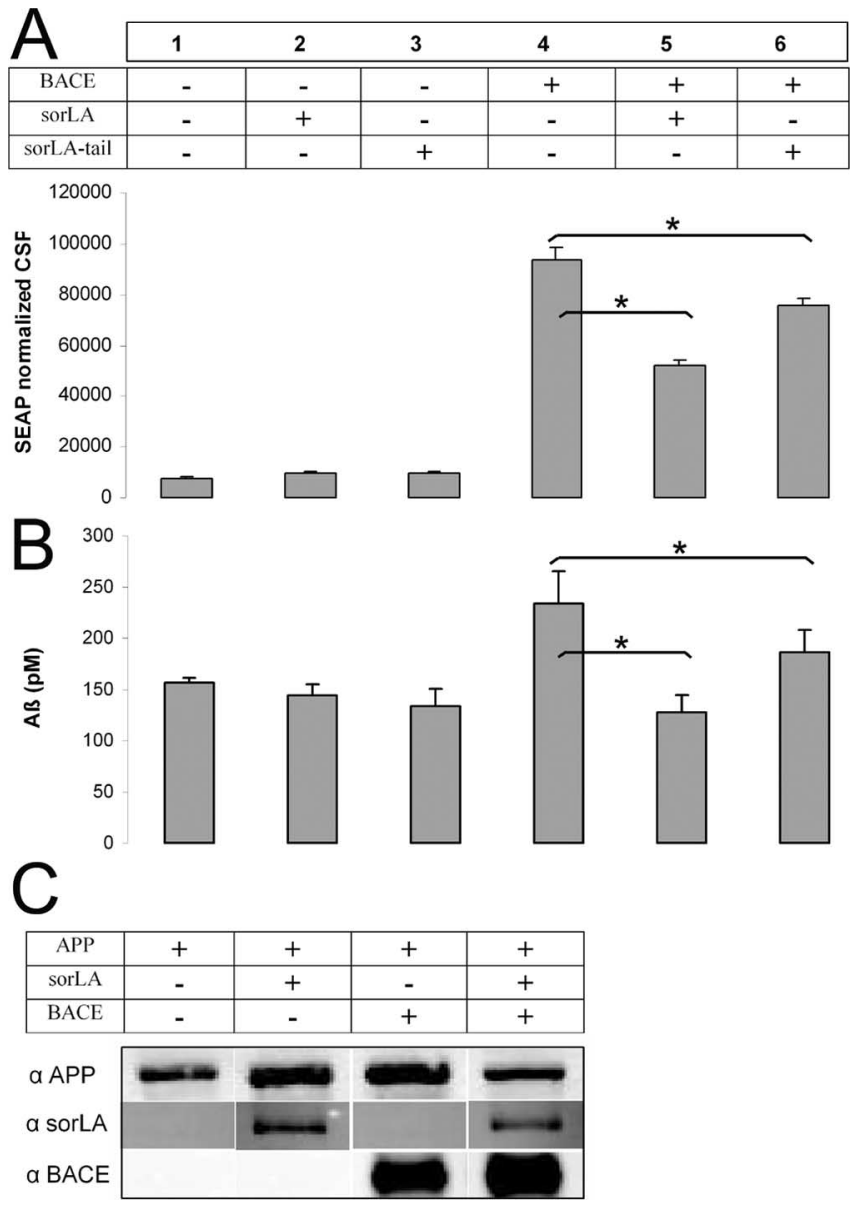

Figure 2. A, APP ectodomain shedding assay shows that APP cleavage is altered by sorLA. HEK293 cells were transfected with SEAP-APP, $\beta$-gal reporter construct, and empty vector ( 1 , $4)$, sorLA $(2,5)$, or sorLA-tail $(3,6)$ constructs with or without BACE. SEAP-APP cleavage is quantitated by alkaline phosphatase activity in conditioned media normalized to $\beta$-gal activity. The results are representative of data of three independent assays. The means and SD of one assay performed in triplicate are shown. ${ }^{*} p<0.05$, Student's $t$ test. $\boldsymbol{B}$, Production of secreted $A \beta$ is dependent on sorLA. 7W CHO cells stably expressing APP were transfected with empty vector (1), sorLA (2), sorLA-tail (3) construct, or BACE (4), BACE-sorLA (5), BACE-sorLA-tail (6). The amount of secreted $A \beta 40$ was measured in triplicate by ELISA assay, and means and SD are given. The experiment was performed in three independent assays $\left({ }^{*} p<0.05\right.$; Student's $t$ test). C, Western blotting of sorLA, APP, and BACE. Cotransfection of APP- empty vector, APPsorLA, APP-BACE-V5, or APP-BACE-V5-sorLA revealed no influence of sorLA expression on APP or BACE levels. Conditions were probed with $8 \mathrm{E} 5$ anti-APP, ms anti-V5, and sorLA antibody as indicated by immunoreactive bands.

dogenous BACE levels. Moreover, the sorLA-tail construct significantly lowered SEAP activity as well, although less efficiently than the full-length construct, which points toward a partial function of the construct in reducing APP cleavage (Fig. $2 A$, lane 6). In addition, overexpression of sorLA did not alter cellular levels of APP or BACE, suggesting that the effect is on APP-BACE trafficking and interactions rather than synthesis or degradation (Fig. 2C).

\section{sorLA reduces secreted $A \beta 40$ dependent on $B A C E$}

The finding that sorLA and sorLA tail both reduce APP cleavage in the presence of BACE suggests that sorLA impacts $\beta$-cleavage. We therefore hypothesized that sorLA also has a BACEdependent effect on $A \beta$ generation. As expected, BACE transfection leads to a significant increase in $\mathrm{A} \beta$ production (Fig. $2 \mathrm{~B}$, lane 4). Cotransfection of sorLA or sorLA tail with BACE blocks this increase and brings $\mathrm{A} \beta$ back to baseline levels, suggesting that sorLA has an important function in regulating APP-BACE interactions (Fig. $2 B$, lanes 5 and 6). Interestingly, overexpression of sorLA or sorLA tail, without BACE overexpression (Fig. $2 B$, lanes 2 and 3), did not lead to an observable reduction in baseline $A \beta$ generation in $\mathrm{CHO}$ cells.

\section{Colocalization of sorLA and C99}

Based on the finding that sorLA tail alone was sufficient to reduce APP cleavage and A $\beta$ generation, we investigated a potential second interaction site between the C-terminal tails of sorLA and APP. In our previous study, we demonstrated the interaction of the N-terminal domains of APP and sorLA (Andersen et al., 2005). To characterize a potential C-terminal interaction site between APP and sorLA, we analyzed whether C99, which lacks most of the extracellular domain of APP, still colocalizes with sorLA. To this end, sorLA and C99-myc-cotransfected N2a (Fig. $3 A$ ) or HEK (Fig. $3 B$ ) cells were immunostained with sorLA rabbit tail antibody, visualized by Alexa 488, and ms 13G8 anti-APP C-terminal antibody labeled by Cy3. The double immunostaining revealed colocalization mainly in the perinuclear region. More distal compartments showed less congruent staining for sorLA and C99. There was no difference in the staining pattern between the individual overexpression of sorLA and C99 and the coexpression of both proteins. This pattern of colocalization was identical to that observed with sorLA and full-length APP.

\section{Interaction of sorLA and C99 in pull-down experiments}

To analyze whether sorLA and C99 bind to each other, extracts from C99-myc and sorLA-transfected N2a cells were pulled down with myc-binding beads and probed with rabbit antisorLA and mouse 9E10 anti-myc antibody. C99 constructs were used to investigate a specific C-terminal tail interaction, because immunoprecipitation of full-length sorLA with APP might reflect the previously described N-terminal domain interaction. Immunoreactive bands of $\sim 250 \mathrm{kDa}$ for sorLA and $20 \mathrm{kDa}$ for C99 were detectable in the immunoprecipitated sample, demonstrating that sorLA was pulled down by C99-myc (Fig. 4, lane 1). To check for nonspecific interactions, individually transfected lysates were probed, resulting in the absence of sorLA pull-down by C99-myc only (Fig. 4, lane 2) or in cells only expressing sorLA (Fig. 4, lane 3). Coimmunoprecipitation of sorLA by C99 was confirmed when using lysates from sorLA-GFP- and C99-myctransfected cells and using GFP-binding beads. Cell extracts from cells expressing either C99-myc or sorLA constructs show no specific pull-down bands (data not shown).

Previous studies have shown that the GYENPTY motif in the cytoplasmatic tail of APP is responsible for the binding of adaptor proteins and seems to mediate endocytosis of APP via the adaptor protein Fe65 and low-density lipoprotein receptor (LDLR)related protein (LRP) (Lai et al., 1995). The GYENPTY motif includes the NPXY motif, which is the endocytosis motif in the tail of the LDLR. To investigate whether the binding of C99 to sorLA is dependent on the GYENPTY motif, a mutant construct of C99 was used, in which tyrosine residues were mutated to alanine residues. Remarkably, the C99DGYENPTY-myc mutant construct was also sufficient to pull down sorLA (Fig. 4, lane 4), indicating a GYENPTY-motif-independent interaction of both proteins. For both C99 wild-type and mutant form, the C99 pulldown produced strong immunoreactive bands at $250 \mathrm{kDa}$, the expected size of sorLA. 
Interaction of sorLA and C99 by FLIM analysis in $\mathrm{N} 2$ a cells

To further study the interaction of C99 and sorLA in intact cells and to determine in which subcellular compartments both proteins interact, we performed FLIM in mouse neuronal N2a cells. FLIM is a new fluorescence resonance energy transfer (FRET)-based technique for detecting intermolecular or intramolecular interactions that take place within a distance of 10 $\mathrm{nm}$ or less. The fluorescence lifetime is influenced by the surrounding environment and shortened in the immediate vicinity of a FRET acceptor fluorophore in proportion to the distance between the fluorophores (Berezovska et al., 2003).

First, we analyzed the proximity of the intracellular domains of APP and sorLA by FLIM. N2A cells were transfected with APP695-myc and sorLA constructs and were stained for the intracellular domain of sorLA by the rabbit C-terminal sorLA antibody labeled by Alexa 488 and for APP by mouse 13G8 labeled by Cy3. The double immunostaining showed colocalization of APP and sorLA in perinuclear and distal compartments. For the FLIM analysis, the change in the lifetime of the donor fluorophore Alexa 488 under different experimental conditions was determined. As a negative control, the lifetime of Alexa 488 attached to the C terminus of sorLA was measured in the absence of acceptor (Cy3), which showed lifetimes of $1985 \pm 20$ ps, equivalent to Alexa 488-IgG alone (Fig. 5A). The lifetime was significantly shortened when sorLA labeled by Alexa 488 was costained with Cy3-labeled APP695 to $1844 \pm 123$ ps, indicating FRET attributable to close proximity between sorLA and APP695 (Fig. 5B). The degree of lifetime shortening is displayed in a pseudo-color-coded image, indicating shortening of lifetime in yellow and red, particularly in the perinuclear region and some endosomal-like structures. Equivalent results were obtained when the acceptor and donor fluorophores were exchanged. In cells transfected with only APP, in the absence of acceptor fluorophore (Cy3), lifetimes of $1962 \pm$ 33 ps were detected. The lifetime was shortened to $1881 \pm 62$ ps when cells were coexpressed with sorLA that was labeled with $\mathrm{Cy} 3$ $(n=12 ; p<0.001)$.

Although the C-terminal domains of APP695 and sorLA are in close proximity, their interaction reflected by a shortening in lifetime could be attributable to the interaction of the $\mathrm{N}$-terminal domains, moving the $\mathrm{C}$-terminal domains closer together. To test whether there is an N-terminal-independent interaction of the C-terminal domains of APP and sorLA, FLIM analysis of the proximity between sorLA and C99-myc, which lacks most of the APP extracellular site, was performed. C99-myc and sorLAtransfected $\mathrm{N} 2 \mathrm{a}$ cells were stained by rabbit C-terminal sorLA antibody labeled by Alexa 488 and for C99-myc by mouse antimyc 9E10 antibody, which was labeled by Cy3. The lifetime of Alexa 488 attached to the $C$ terminus of sorLA (1985 \pm 20 ps) was dramatically shortened when cells coexpressed C99-myc, which was labeled with $\mathrm{Cy} 3$, to $1712 \pm 131$ ps (Fig. 5 C, Table 1). To verify the observation of the pull-down experiments, indicating that sorLA binds C99 independent from the GYENPTY motif, we analyzed the proximity of the C99 $\Delta$ GYENPTY-myc mutant construct to sorLA. We measured a lifetime reduction to $1617 \pm 214$
C99
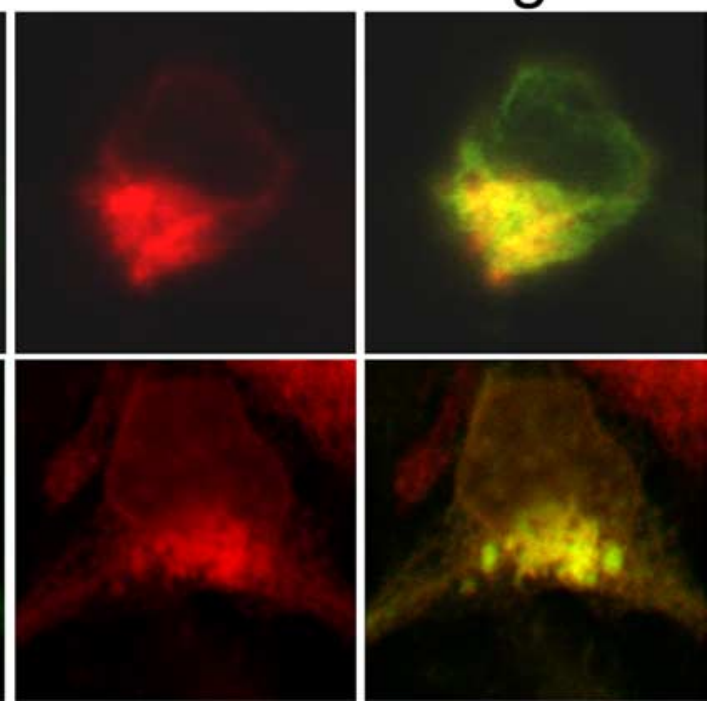

\section{merged}

Figure 3. Localization of sorLA and C 99 in transfected cells. $\boldsymbol{A}, \boldsymbol{B}$, sorLA and C99 were cotransfected into N2a cells (A) or HEK293 cells $(\boldsymbol{B})$ and immunostained with anti-tail sorLA antibody visualized by Alexa 488 (green) and mouse $13 \mathrm{G} 8$ antibody labeled by Cy3 (red). The panels on the right show colocalization of sorLA and C99 in yellow.

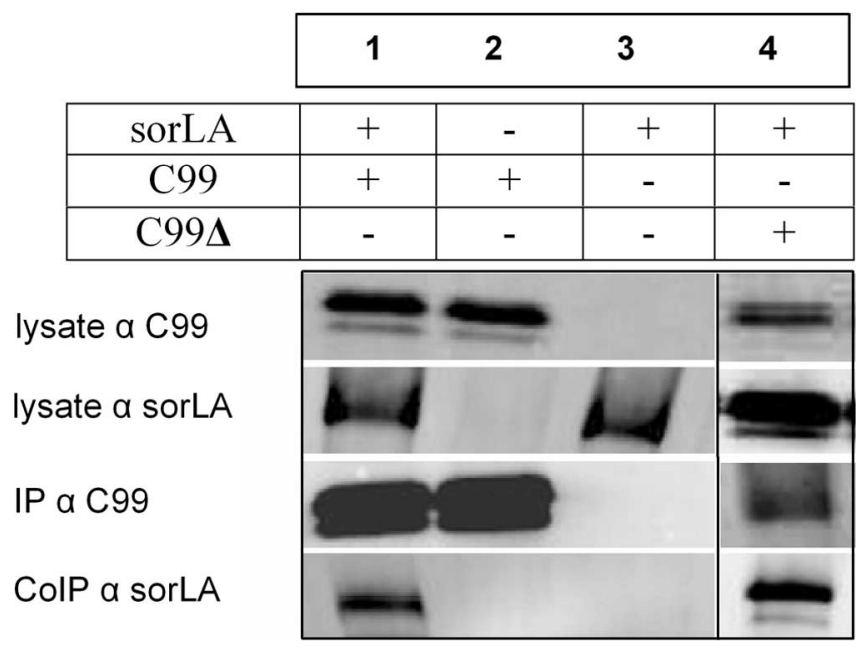

Figure 4. Coimmunoprecipitation of $(99$ and sorLA. C99-myc was pulled down by mycbeads and probed for sorLA by LA-sorLA antibody (lane 1). N2a cells transfected with C99-myc (lane 2) or transfected with sorLA only (lane 3), showed no nonspecific pull-down. Specific coimmunoprecipitation of sorLA was also observed when immunoprecipitating transfected (99DGYENPTY-myc mutant construct (lane 4). For all experiments, specific bands for (99-myc stained with $13 \mathrm{G} 8$ antibody or sorLA were shown from cell lysates and pull-down.

in sorLA-C99DGYENPTY-myc-transfected cells, equivalent to the results of C99 and sorLA interaction (Fig. 5D, Table 1).

Together, our FLIM results confirm the existence of a GYENPTY-independent C-terminal binding site on sorLA that allows for the interaction with the APP C terminus in addition to the interaction domains located in the extracellular region of the proteins (Andersen et al., 2005). Our findings furthermore show that the interaction between sorLA and C99 occurs mainly in the perinuclear region, most likely in the Golgi apparatus.

The sensitivity of the lifetime measurements to proximity is illustrated by an additional negative control. Fluorophores located in proteins on opposite sides of a membrane are usually considered to be too far apart to show FRET. The lifetime of sorLA labeled at the extracellular $\mathrm{N}$ terminus (rabbit anti-sorLA) 


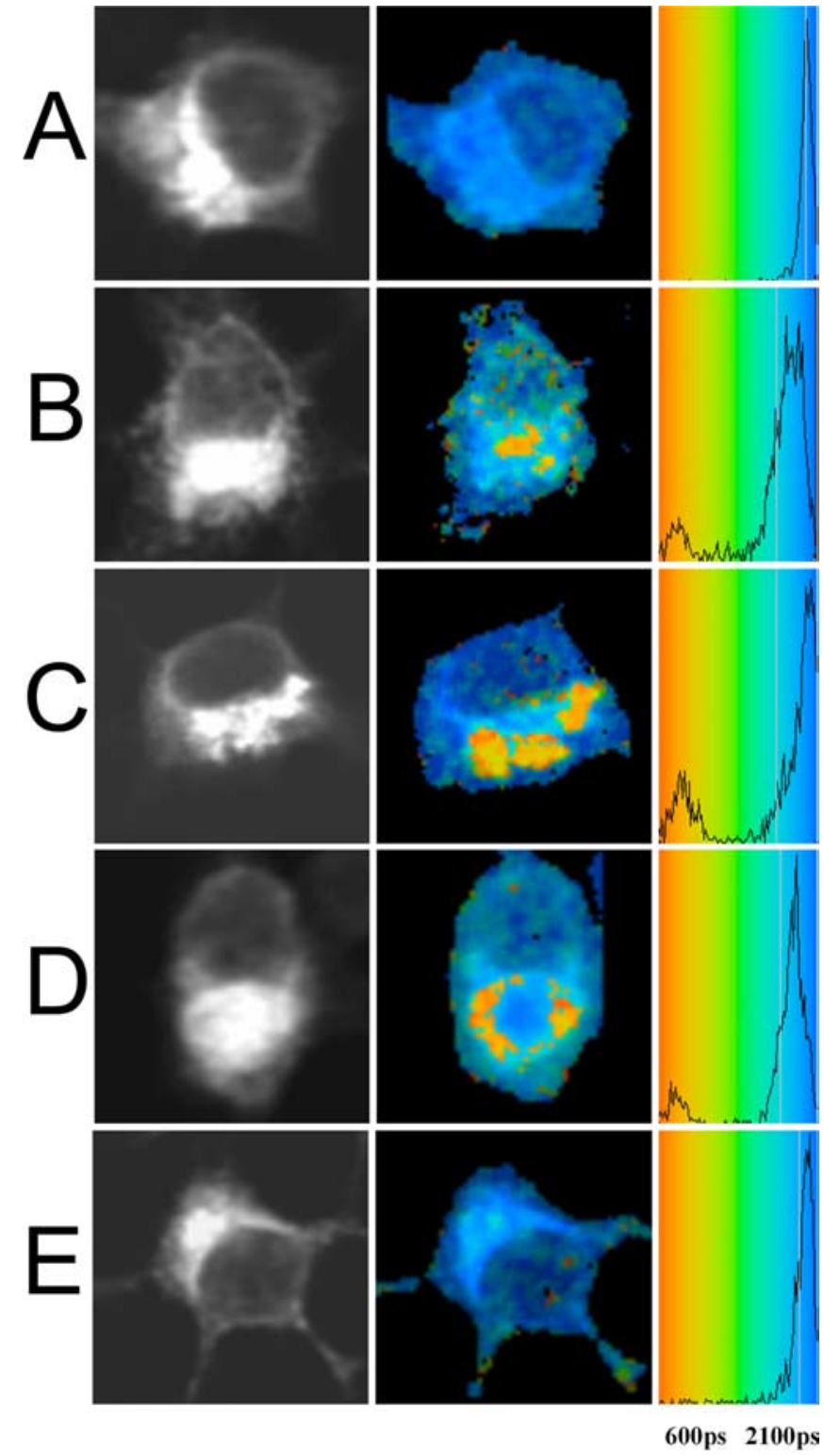

Figure 5. FLIM analysis of sorLA APP and sorLA $C 99$ interaction within N2a cells. $\boldsymbol{A}$, For the negative control, cells were transfected with sorLA and stained by C-terminal sorLA antibody labeled by Alexa 488. The left panel shows confocal images of cells stained for sorLA by donor fluorophore Alexa 488. The color-coded FLIM image highlights the lifetime of the donor fluorophore in picoseconds. $\boldsymbol{B}$, FLIM analysis of sorLA C terminus labeled by Alexa 488 and APP C terminus labeled by Cy3-transfected cells demonstrate shortening of donor lifetime, particularly in the perinuclear region. C, D, N2a cells cotransfected with sorLA, C99-myc (C), or C99DGYENPTY-myc mutant construct $(\boldsymbol{D})$ were labeled on the cytoplasmatic domain by Alexa 488 or by $C y 3$. Shortening of lifetime appeared mainly in the perinuclear region as indicated by red and orange colors in the pseudocolored image. $\boldsymbol{E}$, Cells stained for the $\mathrm{N}$-terminal domain of sorLA by Alexa 488 and C-terminal domain of APP by Cy3 do not show a shortening of donor fluorophore lifetime.

in the presence of APP labeled at the intracellular C terminus with mouse anti-APP antibody $13 \mathrm{G} 8$ labeled by Cy3 was determined (Fig. 5E). The fact that in this control, Alexa 488 lifetime (1938 \pm $45 ; n=9$ ) is close to the negative control, demonstrates that although sorLA and APP colocalize at the light level, the labeled epitopes are too far apart to support FRET. This proves that a change in the fluorescence lifetime, indicating FRET, is a more sensitive measure of proximity than simple colocalization.
Interaction of sorLA and APP by FLIM analysis in primary neurons

To examine the functional relevance of the interaction between sorLA and APP, we furthermore investigated its presence in primary neurons on the level of endogenous protein by our $\mathrm{N}$ - and C-terminal FLIM assay, respectively. Primary neurons were stained for APP by N-terminal 8E5 antibody labeled with Alexa 488 and for sorLA by rabbit SOL-N-terminal antibody labeled with $\mathrm{Cy} 3$. In the absence of an acceptor fluorophore, the donor lifetime was $2167 \pm 21$ (Fig. 6A, Table 2). The lifetime of Alexa 488 attached to the $\mathrm{N}$ terminus of APP was significantly shortened when cells were also stained for sorLA labeled with Cy3, to $1919 \pm 107$ ps (Fig. 6 B, Table 2), indicative of close proximity between the two fluorophores. This was confirmed when the acceptor and donor fluorophores were exchanged, because cells stained for sorLA only showed a lifetime of $2059 \pm 18$ ps, whereas the lifetime was reduced to $1788 \pm 99$ ps when APP was labeled by Cy3 (data not shown; $n=10 ; p<0.001$ ). Because we previously identified the interaction of the C-terminal domains of sorLA and APP in transfected cells, we labeled the sorLA C terminus with Alexa 488 and the APP cytosolic tail with Cy3 on the endogenous proteins. Again, we observed a significant shortening of the donor lifetime to $1985 \pm 47$ ps (Fig. 6C, Table 2), indicating that the C-terminal interaction between sorLA and APP takes place on an endogenous level as well, consistent with our observation that endogenous sorLA and APP can be coimmunoprecipitated from neurons (Andersen et al., 2005). Our data provide strong evidence for the physiological relevance of the interaction between APP and sorLA and show that both proteins come into close proximity in the perinuclear compartments.

\section{Coimmunoprecipitation of sorLA and BACE}

When we analyzed APP cleavage in a shedding assay and measured secreted $A \beta$, we found that both pathways were only inhibited by sorLA when BACE is overexpressed. These results suggest that sorLA impacts BACE activity. We therefore tested the hypothesis that sorLA and BACE interact. Immunohistochemical staining showed colocalization of both proteins in Golgi compartments, as expected from previous studies of BACE localization (von Arnim et al., 2004). Therefore, we asked whether sorLA might be able to interact not only with APP but also with BACE. To analyze BACE-sorLA interactions, we immunoprecipitated sorLA and probed with BACE antibody from cotransfected N2a cells (Fig. 7A). In addition, the complementary coimmunoprecipitation was done, and immunoprecipitated BACE was probed for sorLA (Fig. 7B). Both experiments showed immunoreactive bands for sorLA at $250 \mathrm{kDa}$ and double bands of $\sim 65 \mathrm{kDa}$ in the BACE pull-down or only a single band for BACE in the sorLA pull-down, indicating interaction between sorLA and immature BACE. BACE is present in cells in a partially glycosylated immature and a fully glycosylated mature form. The fact that, in the sorLA pull-down, only the smaller immature BACE was coimmunoprecipitated confirms our hypothesis that sorLA interacts with BACE predominantly in the Golgi, in which immature $\mathrm{BACE}$ resides. To test whether the interaction occurred with endogenous levels of both proteins, we immunoprecipitated endogenous BACE from mouse brain tissue and looked for the coimmunoprecipitation of sorLA by rabbit SOL-sorLA antibody. Control precipitate from samples without BACE antibody showed no immunoreactive bands for sorLA (Fig. 7D), whereas sorLA detection from coimmunoprecipitate demonstrate an endogenous interaction of sorLA and BACE (Fig. 7C). Thus, it is 
possible that APP and BACE compete for sorLA binding within the Golgi.

\section{BACE-APP interaction is reduced by sorLA}

Our results demonstrated the interaction of sorLA and BACE by coimmunoprecipitation. In addition, we found that APP cleavage and $\mathrm{A} \beta$ production were only significantly reduced in HEK293 and CHO cells when BACE is overexpressed. Because these findings suggest a direct impact of sorLA on BACE-APP interaction, we investigated the subcellular distribution of the interaction between BACE and APP in the presence or absence of sorLA. To this end, we used a previously established FLIM assay in mouse neuronal N2a cells transfected with BACE and APP in the presence and absence of sorLA. BACE was stained with Alexa 488-labeled anti-N-terminal BACE antibody and APP by Cy3-labeled 8E5 antibody. Immunostaining showed a typical localization of BACE and APP in the endoplasmic reticulum (ER), Golgi and endosomal-like compartments. As a negative control, lifetime of Alexa 488 attached to the $\mathrm{N}$ terminus of BACE was determined in the absence of acceptor (Cy3) and showed lifetimes of $2051 \pm 17$ ps (Fig. 8A). When Cy3 was attached to the N-terminal domain of APP, we observed a significant decrease in the lifetime to $1939 \pm 59$ ps (Fig. 8 B, Table 3 ). The interaction of BACE and APP was particularly strong in the perinuclear region as indicated by red and orange colors in the pseudocolored images. In contrast, the interaction of BACE and APP was dramatically reduced when we analyzed FRET in the presence of sorLA (Fig. 8C). The lifetime of Alexa 488 attached to the $\mathrm{N}$ terminus of BACE was not significantly different from the negative control $(2027 \pm 28 \mathrm{ps})$, indicating that sorLA inhibits the interaction of BACE with APP. Examination of the FLIM images suggest that APP-BACE interaction was specifically disrupted in the Golgi apparatus. These results suggest that sorLA affected the interaction but not the subcellular distribution of APP or BACE (Fig. 8, intensity images); both were present in the Golgi, in which the presence of sorLA affected their close association.

\section{Discussion}

The potential importance of SorLA in the pathophysiology of Alzheimer's disease is suggested by the observations that sorLA expression is dramatically decreased in the brain of Alzheimer patients and that sorLA null mice have accelerated amyloid production (Scherzer et al., 2004; Andersen et al., 2005). In this study, we demonstrate that the putative sorting receptor sorLA has a complex set of interactions with APP and, surprisingly, BACE. In addition to the previously described N-terminal interaction between sorLA and APP (Andersen et al., 2005), we identify a new functional C-terminal binding site on sorLA for APP. sorLA overexpression reduces the $\beta$-cleavage of APP and, in consequence, also inhibits $\mathrm{A} \beta$ generation. Our data suggest that sorLA interacts with BACE in the Golgi, in which it appears to directly inhibit BACE-APP complex formation and thereby reduces this component of BACE activity on APP. Thus, we find two new interactions of sorLA: with the C terminus of APP and with BACE, which may contribute to the mechanism how sorLA influences APP metabolism and AD.

Recent studies have shown that the Vps10p receptor sorLA is directly involved in the intracellular transport and processing of

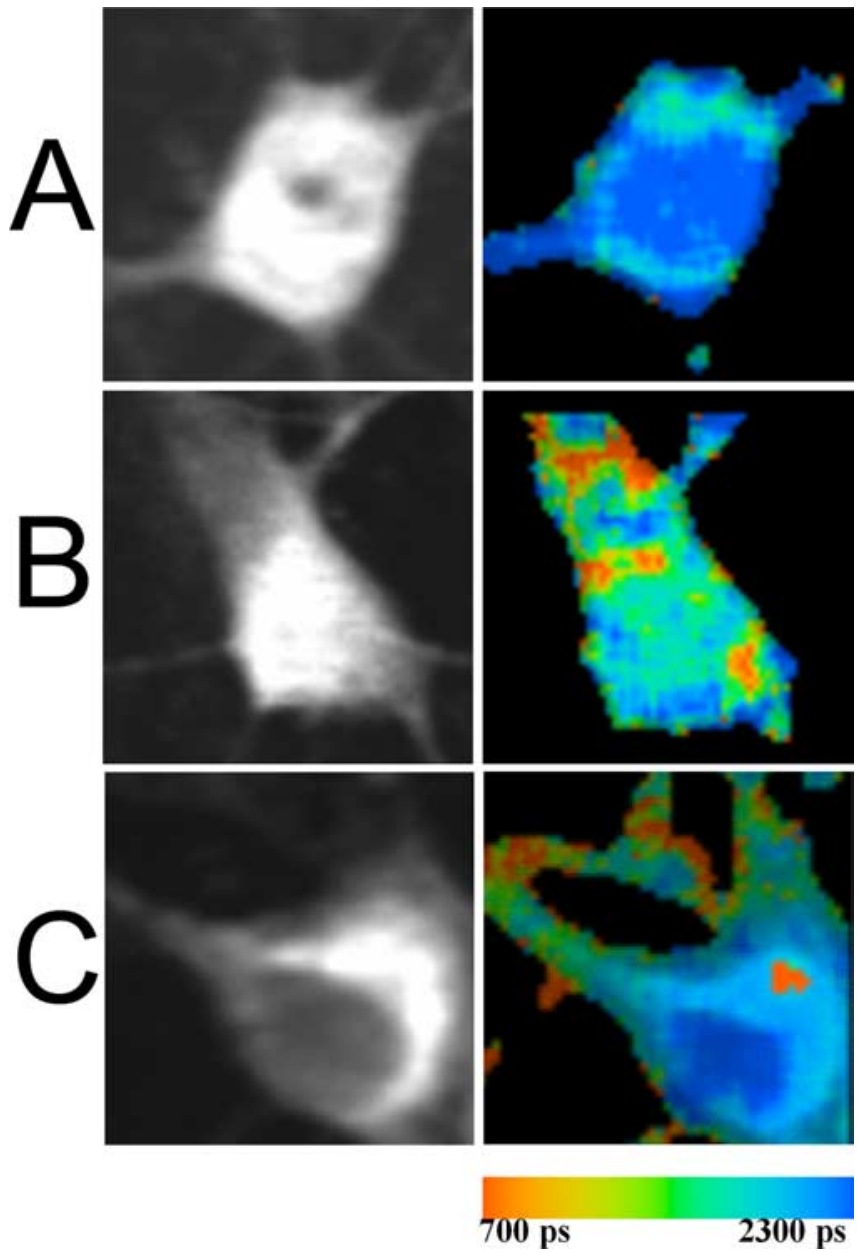

Figure 6. FLIM analysis of APP and sorLA on endogenous proteins. $A-C$, Primary neurons were immunostained for endogenous APP by N-terminal $8 \mathrm{E} 5$ antibody $(A, B)$ or by $C$-terminal 1368 antibody $(\boldsymbol{C})$ labeled by Alexa $488(\boldsymbol{A}, \boldsymbol{B})$ or by $\mathrm{Cy} 3(\boldsymbol{C})$. The lifetime was measured in the presence $(\boldsymbol{B}, \boldsymbol{C})$ or absence $(\boldsymbol{A})$ of acceptor fluorophore $C \mathrm{C} 3$ attached to the $\mathrm{N}$ terminus of endogenous sorLA $(\boldsymbol{B})$ or $\mathrm{C}$-terminal of endogenous APP ( $\boldsymbol{C}$ ). Pseudocolored FLIM images highlight FRET between APP and sorLA in juxtanuclear and distal compartments $(\boldsymbol{B}, \boldsymbol{C})$.

APP (Andersen et al., 2005) and that the overexpression of sorLA results in reduced cell surface APP and decreased levels of secreted sAPP and A $\beta$. These results raised the question of whether sorLA could mediate the internalization of cell surface APP and thereby also reduce the processing of APP into sAPP and A $\beta$. It has been shown that sorLA is capable of mediating endocytosis (Jacobsen et al., 2001) and binds apolipoprotein E-rich lipoproteins and mediate their uptake (Taira et al., 2001). When we 
Table 2. APP sorLA FLIM analysis on endogenous proteins in primary neurons

\begin{tabular}{lllll}
\hline Donor & Acceptor & $\begin{array}{l}\text { Lifetime }(\mathrm{ps}) \\
\text { (mean } \pm \text { SD) }\end{array}$ & $n$ (cells) & $\begin{array}{l}\text { Significance } \\
\text { (compared with control) }\end{array}$ \\
\hline APP-N (Alexa488) & None & $2167 \pm 21$ & 12 & \\
APP-N (Alexa488) & sorLA-N (Cy3) & $1919 \pm 107$ & 14 & $p<0.001$ \\
APP-N (Alexa488) & None & $2230 \pm 15$ & 6 & $p<0.001$ \\
APP-N (Alexa488) & sorLA-N (Cy3) & $2136 \pm 42$ & 8 & \\
sorLA-N (Alexa488) & None & $2059 \pm 18$ & 6 & $p<0.001$ \\
sorLA-N (Alexa488) & APP-N (Cy3) & $1788 \pm 99$ & 10 & \\
sorLA-C (Alexa488) & None & $2059 \pm 18$ & 6 & $p<0.001$ \\
sorLA-C (Alexa488) & APP-C (Cy3) & $1985 \pm 47$ & 8 &
\end{tabular}

$\mathrm{N}, \mathrm{N}$-terminal; C, C-terminal.

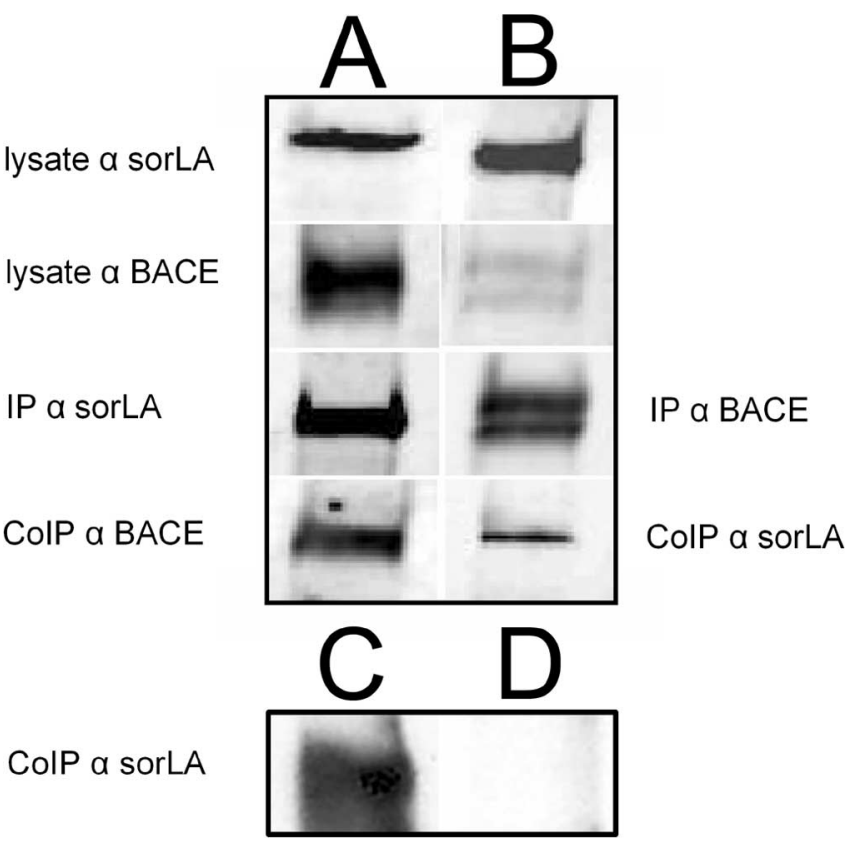

Figure 7. Coimmunoprecipitation of BACE and sorLA. sorLA was immunoprecipitated and probed for BACE by BACE antibody from transfected N2a cell extracts. $\boldsymbol{A}$, Immunoreactive bands of sorLA and BACE in lysates and pull-downs demonstrate BACE-sorLA interaction. $B$, Interaction of sorLA and BACE could be proven when BACE was immunoprecipitated and sorLA was shown to coimmunoprecipitate. Double bands of BACE show mature and immature form of BACE. C, Coimmunoprecipitation of endogenous BACE and sorLA from mouse brain extract. BACE was immunoprecipitated with rabbit N-terminal BACE antibody and probed for sorLA. D, In the absence of the pull-down antibody, no immunoreactive band was detected for sorLA. IP, Immunopreciptation; CoIP, coimmunopreciptation.

investigated uptake of APP from the cell surface, however, we found that APP internalization is independent of sorLA. The hypothesis that sorLA does not have a major function in endocytosis is further supported by its low rate of endocytosis when compared with other apoE receptors (Gliemann et al., 2004). Thus, these data extend and support the concept that the reduced level of APP on the cell surface in sorLA-overexpressing cells is achieved by the retention of APP in the Golgi apparatus, rather than an effect on cell surface uptake of APP.

To understand the role of sorLA in APP processing in detail, we investigated the influence of sorLA on APP cleavage and A $\beta$ generation. In addition to the observation that sorLA influenced APP $\beta$-cleavage, we uncovered a new interaction of the sorLA cytoplasmatic tail in APP processing. Our results further indicated a partial effect of the C-terminal sorLA tail on APP cleavage. In line with these results, sorLA and sorLA tail also inhibit $A \beta$ generation in an independent assay and different cell line. These functional data suggested the existence of a second C-terminal interaction site between sorLA and APP.

We recently demonstrated direct binding of the N-terminal domain of sorLA and APP by surface plasmon resonance analysis, analytical ultracentrifugation, and cell culture experiments (Andersen et al., 2005). Herein, we now report a second binding site between the C-terminal domains of sorLA and APP. We show that sorLA coimmunoprecipitates with C99, which lacks most of the extracellular domain of APP. Coexpression of sorLA and C99 showed that both proteins predominantly reside in the Golgi apparatus and endosomal-like compartments. When we used C99 mutation construct of the GYENPTY motif, a motif in the C-terminal tail of APP that has been suggested to mediate endocytosis of APP (Lai et al., 1995), we observed that C99 and sorLA bind to each other independently from the GYENPTY motif and bolster the observation that sorLA has no function in endocytosis of APP. Moreover, we used FLIM, a sensitive FRET-based technique (Berezovska et al., 2003), to analyze complex formation between sorLA and C99. When we attached fluorophores to the C-terminal tails of C99 and sorLA, we observed a strong FRET signal in the perinuclear region and to a lower extent in distal compartments. Our current data also support the presence of the sorLA and APP interaction in intact neurons under physiological conditions. Using FLIM analysis, on APP and sorLA in primary neurons, we were able to show that the endogenous proteins interact in the Golgi. This complements and extends our previous observations that sorLA and APP can be coimmunoprecipitated from neuronal cells (Andersen et al., 2005).

A striking result of our current study is the finding that APP cleavage and $\mathrm{A} \beta$ generation in $\mathrm{CHO}$ and HEK293 cells were only reduced by sorLA when BACE, the $\beta$-cleaving enzyme, was overexpressed. Previous results in SY5Y cells with high endogenous BACE levels have shown a sorLA-dependent reduction of sAPP and $\mathrm{A} \beta$ production without overexpressing BACE (Andersen et al., 2005). Herein, we used cell lines with barely detectable BACE levels and overexpressed BACE to highlight the relevance of sorLA in BACE-dependent APP processing. Moreover, sorLA interaction with APP and BACE was shown using endogenous levels of protein, indicating that the results we describe are not a consequence of protein overexpression.

We hypothesized that sorLA impacts a subset of APP-BACE interactions and analyzed whether the sorLA effects could be localized to a specific subcellular compartment. Several lines of data are consistent with a predominant effect of sorLA on APP and BACE in the Golgi. APP and BACE colocalize in the Golgi apparatus and ER (Huse et al., 2000). The acidic $\mathrm{pH}$ optimum of the $\beta$-cleavage suggests that this step in APP processing predominates within late Golgi apparatus compartments or endosomal compartments (Sinha and Lieberburg, 1999; Sinha et al., 1999; Vassar et al., 1999; Lin et al., 2000) and is consistent with previous findings that $\beta$-secretase cleavage occurs in all of these compartments (Haass et al., 1992; Koo and Squazzo, 1994; Koo et al., 1996; Perez et al., 1996).

sorLA is strongly expressed in the Golgi, in which it appears to interact with APP, as suggested by both FLIM studies and the observation that sorLA and APP are mainly colocalized in the same subcellular fraction containing markers of the cis-Golgi (Andersen et al., 2005). Moreover, our FLIM studies suggest that 
BACE and APP interaction is disrupted by sorLA primarily in the perinuclear region. Because the reduced APP-BACE complex formation in sorLA-overexpressing cells is not attributable to altered levels of APP and BACE or to a shift in the subcellular distribution of BACE, we postulate that sorLA might have a direct effect on BACE. Our current study provides evidence for the presence of a complex containing both sorLA and BACE. When BACE and sorLAcotransfected cells or endogenous proteins from mouse brain extracts were immunoprecipitated, coimmunoprecipitation of BACE using anti-sorLA antibodies and sorLA using anti-BACE antibodies could be demonstrated. Because we mainly observe an interaction between sorLA, APP, and $\mathrm{BACE}$ in the perinuclear region, it seems likely that sorLA particularly affects APP processing and thereby $A \beta$ generation in the ER and Golgi apparatus. In fact, $\mathrm{A} \beta$ can be generated in the ER and Golgi apparatus (Kuentzel et al., 1993; Chyung et al., 1997; Cook et al., 1997; Hartmann et al., 1997).

In conclusion, we envision a model in which sorLA impacts APP $\beta$-cleavage and $\mathrm{A} \beta$ production by influencing complex formation between APP and BACE predominantly in the Golgi apparatus (Fig. 9, schematic overview). According to this model, sorLA mediates transport of APP through the Golgi and, possibly by blocking access of BACE to APP, inhibits APP $\beta$-site cleavage. Because full-length sorLA is more efficient than the sorLA C-terminal tail in this respect, it is possible that interactions between the luminal domain of sorLA and the luminal domain of APP [demonstrated by Andersen et al. (2005)] contribute to protecting it from BACE cleavage. This model predicts that sorLA-mediated retention of APP in the Golgi and protection from BACE cleavage plays an important regulatory role in determining the amount of precursor that is released into the later secretory pathway for proteolytic processing into $\mathrm{A} \beta$.

Moreover, we also found evidence of an interaction of sorLA with BACE. sorLA and BACE mainly colocalized in the Golgi, suggesting that sorLA also binds BACE in the Golgi and thereby inhibits its access to APP. Although it is also possible that sorLA contributes to retrograde transport of BACE to the Golgi, the observation that sorLA coimmunoprecipitates principally with nonglycosylated immature forms of BACE points to an interaction in the Golgi.

The observations that sorLA is downregulated in AD brain and that, in experimental systems, sorLA reduction and overexpression impact $A \beta$ production point to a pivotal role of sorLA in AD pathophysiology (Andersen et al., 2005). Although the sorLA-APP interactions we report here have similarities to the in-

n.S., Nonsignificant.

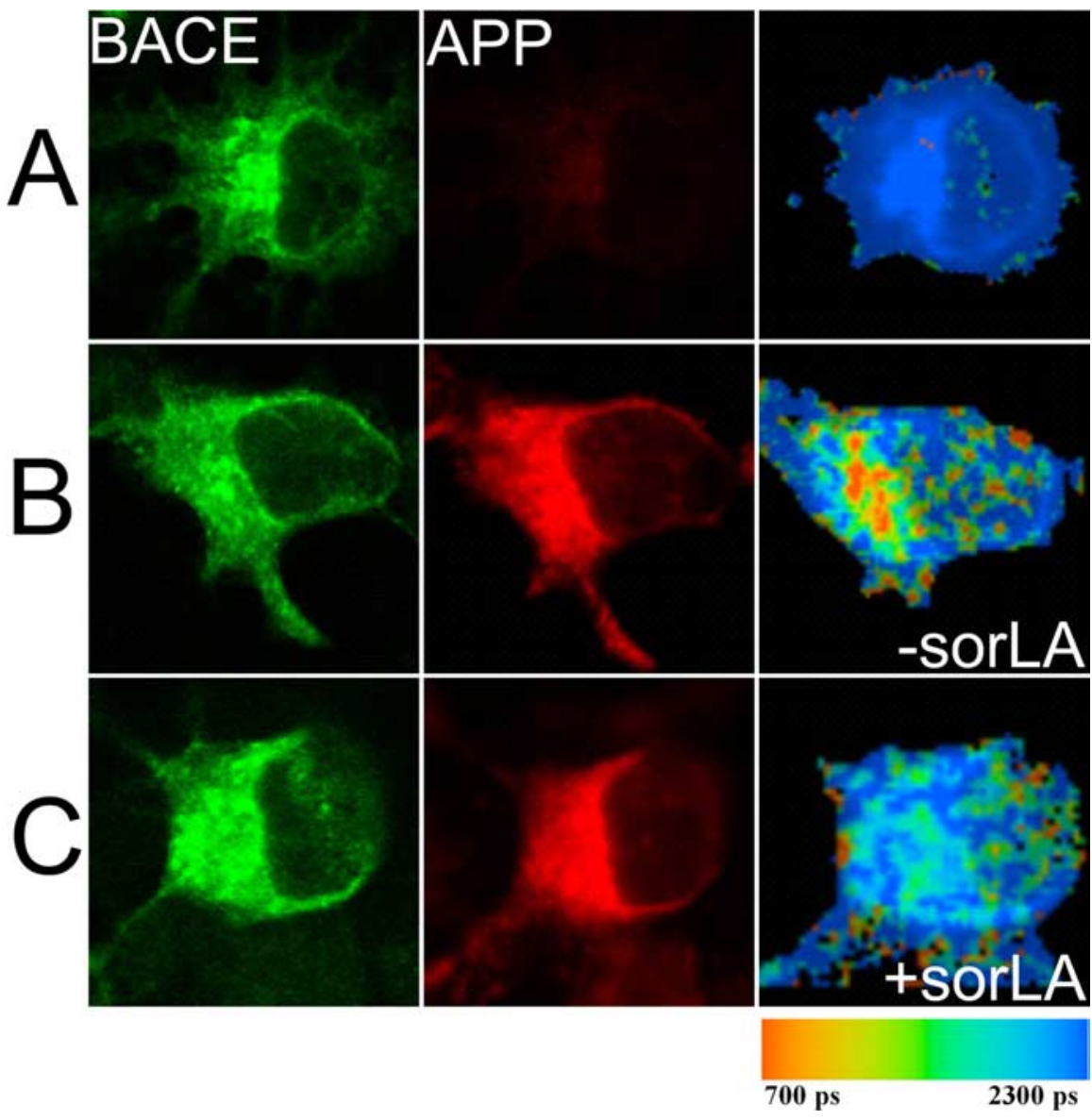

Figure 8. Interaction of APP and BACE dependent on sorLA. For FLIM analysis of APP and BACE proximity, N2a cells were transfected with BACE, BACE-APP, and BACE-APP-sorLA. BACE was labeled on the N-terminal domain by a BACE antibody labeled by Alexa 488 and APP by N-terminal $8 \mathrm{E} 5$ antibody labeled by $C y 3$. $A$, In the absence of acceptor fluorophore Cy3, lifetime averages at $2051 \pm 17$ ps. $\boldsymbol{B}, \boldsymbol{C}$, BACE-APP-transfected cells show BACE-APP interaction mainly in the perinuclear region $(\boldsymbol{B})$, whereas the FRET interaction in cells that also expressed sorLA was strongly reduced $(\boldsymbol{C})$. Intensity images of BACE-APP and BACE-APP-sorLA-transfected cells revealed that the immunostaining pattern for BACE or APP is not significantly altered by sorLA.

Table 3. FLIM assay for BACE-APP interaction in N2a cells

\begin{tabular}{|c|c|c|c|c|c|}
\hline Donor & Acceptor & sorlA & $\begin{array}{l}\text { Lifetime (ps) } \\
\text { (mean } \pm \text { SD) }\end{array}$ & $n$ (cells) & $\begin{array}{l}\text { Significance } \\
\text { (compared with control) }\end{array}$ \\
\hline BACE (Alexa 488) & None & & $2035 \pm 18$ & 8 & \\
\hline BACE (Alexa 488) & APP-myc (Cy3) & & $1756 \pm 184$ & 10 & $p<0.001$ \\
\hline BACE (Alexa 488) & APP-myc (Cyз) & + sorlA & $2022 \pm 65$ & 10 & n.s. \\
\hline BACE (Alexa 488) & None & & $2051 \pm 17$ & 9 & \\
\hline BACE (Alexa 488) & APP-myc (Cy3) & & $1939 \pm 59$ & 9 & $p<0.001$ \\
\hline BACE (Alexa 488) & APP-myc (Cy3) & + sorlA & $2027 \pm 28$ & 12 & n.s. \\
\hline BACE (Alexa 488) & None & & $1996 \pm 43$ & 10 & \\
\hline BACE (Alexa 488) & APP-myc (Cy3) & & $1902 \pm 48$ & 10 & $p<0.001$ \\
\hline BACE (Alexa 488) & APP-myc (Cyз) & + sorlA & $2019 \pm 35$ & 9 & n.s \\
\hline
\end{tabular}

teraction of the LRP with APP, which also interacts through both C-terminal and luminal domain interactions (Kinoshita et al., 2003), the lack of effect of the NPXY mutant on APP-sorLA interactions argue that the adaptor proteins mediating the interaction must be different from the NPXY motif interacting adaptor Fe65. The identity of these postulated adaptor proteins is uncertain. For example, although GGA proteins are known to interact with both BACE and sorLA (Jacobsen et al., 2002; Shiba et al., 2004; von Arnim et al., 2004; He et al., 2005), GGA is monomeric, and both interactions are believed to be mediated via the VHS (Vps27, Hrs, and signal transducing adaptor mole- 


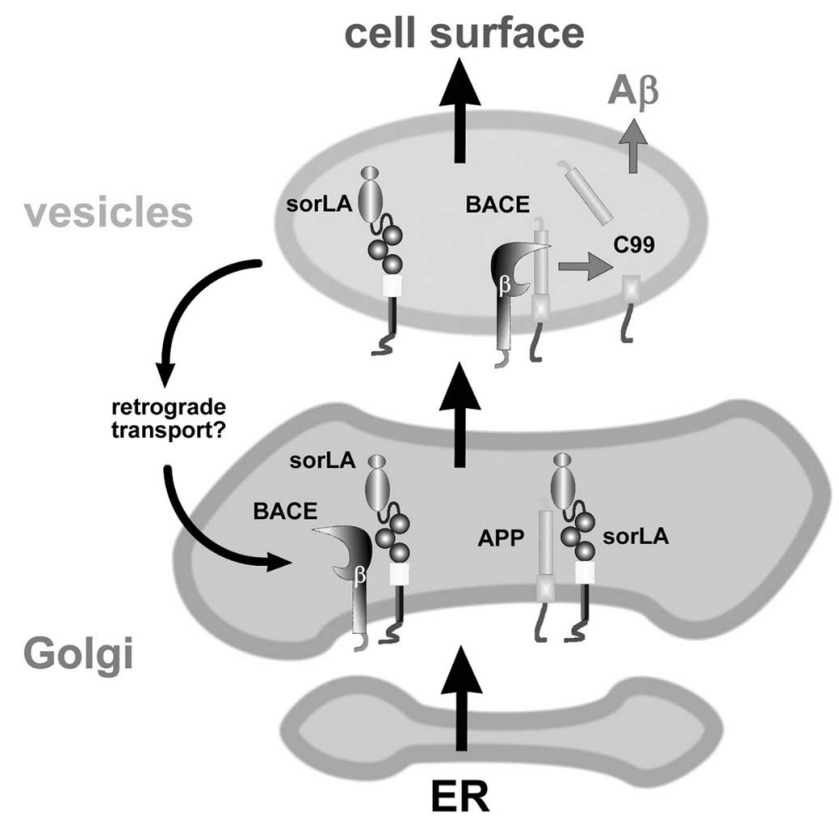

Figure 9. Schematic overview of how sorLA influences APP and BACE interaction. We hypothesize that sorLA regulates trafficking of APP through the Golgi, mainly through slowing release of the precursor to the cell surface and blocking interaction with BACE. Only those APP molecules that are released by sorLA are accessible for proteolytic cleavage either in the Golgi or distal cellular compartments.

cule) domain, arguing against a mechanism through which GGA interacts with both BACE and sorLA simultaneously. Additional evaluation of the molecular mechanisms of sorLA-APP and sorLA-BACE interactions may lead to insight into APP processing and its alterations in Alzheimer's disease.

\section{References}

Andersen OM, Reiche J, Schmidt V, Gotthardt M, Spoelgen R, Behlke J, von Arnim CA, Breiderhoff T, Jansen P, Wu X, Bales KR, Cappai R, Masters CL, Gliemann J, Mufson EJ, Hyman BT, Paul SM, Nykjaer A, Willnow TE (2005) Neuronal sorting protein-related receptor sorLA/LR11 regulates processing of the amyloid precursor protein. Proc Natl Acad Sci USA 102:13461-13466.

Bacskai BJ, Skoch J, Hickey GA, Allen R, Hyman BT (2003) Fluorescence resonance energy transfer determinations using multiphoton fluorescence lifetime imaging microscopy to characterize amyloid- $\beta$ plaques. J Biomed Opt 8:368-375.

Berezovska O, Bacskai BJ, Hyman BT (2003) Monitoring proteins in intact cells. Sci Aging Knowledge Environ 2003:PE14.

Chyung AS, Greenberg BD, Cook DG, Doms RW, Lee VM (1997) Novel $\beta$-secretase cleavage of beta-amyloid precursor protein in the endoplasmic reticulum/intermediate compartment of NT2N cells. J Cell Biol 138:671-680.

Cook DG, Forman MS, Sung JC, Leight S, Kolson DL, Iwatsubo T, Lee VM, Doms RW (1997) Alzheimer's A beta(1-42) is generated in the endoplasmic reticulum/intermediate compartment of NT2N cells. Nat Med 3:1021-1023

Fukumoto H, Tomita T, Matsunaga H, Ishibashi Y, Saido TC, Iwatsubo T (1999) Primary cultures of neuronal and non-neuronal rat brain cells secrete similar proportions of amyloid beta peptides ending at A $\beta 40$ and A $\beta 42$. NeuroReport 10:2965-2969.

Fukumoto H, Tennis M, Locascio JJ, Hyman BT, Growdon JH, Irizarry MC (2003) Age but not diagnosis is the main predictor of plasma amyloid $\beta$-protein levels. Arch Neurol 60:958-964.

Gliemann J, Hermey G, Nykjaer A, Petersen CM, Jacobsen C, Andreasen PA (2004) The mosaic receptor sorLA/LR11 binds components of the plasminogen-activating system and platelet-derived growth factor-BB similarly to LRP1 (low-density lipoprotein receptor-related protein), but mediates slow internalization of bound ligand. Biochem J 381:203-212.

Haass C, Koo EH, Mellon A, Hung AY, Selkoe DJ (1992) Targeting of cell- surface $\beta$-amyloid precursor protein to lysosomes: alternative processing into amyloid-bearing fragments. Nature 357:500-503.

Hartmann T, Bieger SC, Bruhl B, Tienari PJ, Ida N, Allsop D, Roberts GW, Masters CL, Dotti CG, Unsicker K, Beyreuther K (1997) Distinct sites of intracellular production for Alzheimer's disease A $\beta 40 / 42$ amyloid peptides. Nat Med 3:1016-1020.

He X, Li F, Chang WP, Tang J (2005) GGA proteins mediate the recycling pathway of memapsin 2 (BACE). J Biol Chem 280:11696-11703.

Huse JT, Pijak DS, Leslie GJ, Lee VM, Doms RW (2000) Maturation and endosomal targeting of $\beta$-site amyloid precursor protein-cleaving enzyme. The Alzheimer's disease $\beta$-secretase. J Biol Chem 275:33729-33737.

Hussain I, Powell D, Howlett DR, Tew DG, Meek TD, Chapman C, Gloger IS, Murphy KE, Southan CD, Ryan DM, Smith TS, Simmons DL, Walsh FS, Dingwall C, Christie G (1999) Identification of a novel aspartic protease (Asp 2) as $\beta$-secretase. Mol Cell Neurosci 14:419-427.

Irizarry MC, Deng A, Lleo A, Berezovska O, Von Arnim CA, MartinRehrmann M, Manelli A, LaDu MJ, Hyman BT, Rebeck GW (2004) Apolipoprotein E modulates $\gamma$-secretase cleavage of the amyloid precursor protein. J Neurochem 90:1132-1143.

Jacobsen L, Madsen P, Jacobsen C, Nielsen MS, Gliemann J, Petersen CM (2001) Activation and functional characterization of the mosaic receptor SorLA/LR11. J Biol Chem 276:22788-22796.

Jacobsen L, Madsen P, Nielsen MS, Geraerts WP, Gliemann J, Smit AB, Petersen CM (2002) The sorLA cytoplasmic domain interacts with GGA1 and -2 and defines minimum requirements for GGA binding. FEBS Lett 511:155-158.

Kinoshita A, Whelan CM, Smith CJ, Mikhailenko I, Rebeck GW, Strickland DK, Hyman BT (2001) Demonstration by fluorescence resonance energy transfer of two sites of interaction between the low-density lipoprotein receptor-related protein and the amyloid precursor protein: role of the intracellular adapter protein Fe65. J Neurosci 21:8354-8361.

Kinoshita A, Shah T, Tangredi MM, Strickland DK, Hyman BT (2003) The intracellular domain of the low density lipoprotein receptor-related protein modulates transactivation mediated by amyloid precursor protein and Fe65. J Biol Chem 278:41182-41188.

Koo EH, Squazzo SL (1994) Evidence that production and release of amyloid $\beta$-protein involves the endocytic pathway. J Biol Chem 269:17386-17389.

Koo EH, Squazzo SL, Selkoe DJ, Koo CH (1996) Trafficking of cell-surface amyloid $\beta$-protein precursor. I. Secretion, endocytosis and recycling as detected by labeled monoclonal antibody. J Cell Sci 109:991-998.

Kosaka T, Imagawa M, Seki K, Arai H, Sasaki H, Tsuji S, Asami-Odaka A, Fukushima T, Imai K, Iwatsubo T (1997) The $\beta$ APP717 Alzheimer mutation increases the percentage of plasma amyloid- $\beta$ protein ending at $\mathrm{A}$ $\beta 42(43)$. Neurology 48:741-745.

Kuentzel SL, Ali SM, Altman RA, Greenberg BD, Raub TJ (1993) The Alzheimer $\beta$-amyloid protein precursor/protease nexin-II is cleaved by secretase in a trans-Golgi secretory compartment in human neuroglioma cells. Biochem J 295:367-378.

Lai A, Sisodia SS, Trowbridge IS (1995) Characterization of sorting signals in the $\beta$-amyloid precursor protein cytoplasmic domain. J Biol Chem 270:3565-3573.

Lichtenthaler SF, Dominguez DI, Westmeyer GG, Reiss K, Haass C, Saftig P, De Strooper B, Seed B (2003) The cell adhesion protein P-selectin glycoprotein ligand-1 is a substrate for the aspartyl protease BACE1. J Biol Chem 278:48713-48719.

Lin X, Koelsch G, Wu S, Downs D, Dashti A, Tang J (2000) Human aspartic protease memapsin 2 cleaves the $\beta$-secretase site of beta-amyloid precursor protein. Proc Natl Acad Sci USA 97:1456-1460.

Perez RG, Squazzo SL, Koo EH (1996) Enhanced release of amyloid $\beta$-protein from codon 670/671 "Swedish" mutant $\beta$-amyloid precursor protein occurs in both secretory and endocytic pathways. J Biol Chem 271:9100-9107.

Puertollano R, Randazzo PA, Presley JF, Hartnell LM, Bonifacino JS (2001) The GGAs promote ARF-dependent recruitment of clathrin to the TGN. Cell 105:93-102.

Scherzer CR, Offe K, Gearing M, Rees HD, Fang G, Heilman CJ, Schaller C, Bujo H, Levey AI, Lah JJ (2004) Loss of apolipoprotein E receptor LR11 in Alzheimer disease. Arch Neurol 61:1200-1205.

Scheuner D, Eckman C, Jensen M, Song X, Citron M, Suzuki N, Bird TD, Hardy J, Hutton M, Kukull W, Larson E, Levy-Lahad E, Viitanen M, Peskind E, Poorkaj P, Schellenberg G, Tanzi R, Wasco W, Lannfelt L, Selkoe D, et al. (1996) Secreted amyloid $\beta$-protein similar to that in the senile plaques of Alzheimer's disease is increased in vivo by the presenilin 
1 and 2 and APP mutations linked to familial Alzheimer's disease. Nat Med 2:864-870.

Selkoe DJ (2001) Alzheimer's disease: genes, proteins, and therapy. Physiol Rev 81:741-766.

Shiba T, Kametaka S, Kawasaki M, Shibata M, Waguri S, Uchiyama Y, Wakatsuki S (2004) Insights into the phosphoregulation of $\beta$-secretase sorting signal by the VHS domain of GGA1. Traffic 5:437-448.

Sinha S, Lieberburg I (1999) Cellular mechanisms of $\beta$-amyloid production and secretion. Proc Natl Acad Sci USA 96:11049-11053.

Sinha S, Anderson JP, Barbour R, Basi GS, Caccavello R, Davis D, Doan M, Dovey HF, Frigon N, Hong J, Jacobson-Croak K, Jewett N, Keim P, Knops J, Lieberburg I, Power M, Tan H, Tatsuno G, Tung J, Schenk D, et al. (1999) Purification and cloning of amyloid precursor protein $\beta$-secretase from human brain. Nature 402:537-540.

Taira K, Bujo H, Hirayama S, Yamazaki H, Kanaki T, Takahashi K, Ishii I, Miida T, Schneider WJ, Saito Y (2001) LR11, a mosaic LDL receptor family member, mediates the uptake of ApoE-rich lipoproteins in vitro. Arterioscler Thromb Vasc Biol 21:1501-1506.

Takatsu H, Katoh Y, Shiba Y, Nakayama K (2001) Golgi-localizing, $\gamma$-adaptin ear homology domain, ADP-ribosylation factor-binding (GGA) proteins interact with acidic dileucine sequences within the cytoplasmic domains of sorting receptors through their Vps27p/Hrs/STAM (VHS) domains. J Biol Chem 276:28541-28545.

Vassar R, Bennett BD, Babu-Khan S, Kahn S, Mendiaz EA, Denis P, Teplow DB, Ross S, Amarante P, Loeloff R, Luo Y, Fisher S, Fuller J, Edenson S, Lile J, Jarosinski MA, Biere AL, Curran E, Burgess T, Louis JC, et al.
(1999) $\beta$-Secretase cleavage of Alzheimer's amyloid precursor protein by the transmembrane aspartic protease BACE. Science 286:735-741.

von Arnim CA, Tangredi MM, Peltan ID, Lee BM, Irizarry MC, Kinoshita A, Hyman BT (2004) Demonstration of BACE ( $\beta$-secretase) phosphorylation and its interaction with GGA1 in cells by fluorescence-lifetime imaging microscopy. J Cell Sci 117:5437-5445.

Wolfe MS, Kopan R (2004) Intramembrane proteolysis: theme and variations. Science 305:1119-1123.

Wolfe MS, Xia W, Ostaszewski BL, Diehl TS, Kimberly WT, Selkoe DJ (1999) Two transmembrane aspartates in presenilin-1 required for presenilin endoproteolysis and $\gamma$-secretase activity. Nature 398:513-517.

Xia W, Ray WJ, Ostaszewski BL, Rahmati T, Kimberly WT, Wolfe MS, Zhang J, Goate AM, Selkoe DJ (2000) Presenilin complexes with the C-terminal fragments of amyloid precursor protein at the sites of amyloid $\beta$-protein generation. Proc Natl Acad Sci USA 97:9299-9304.

Yamazaki H, Bujo H, Kusunoki J, Seimiya K, Kanaki T, Morisaki N, Schneider WJ, Saito Y (1996) Elements of neural adhesion molecules and a yeast vacuolar protein sorting receptor are present in a novel mammalian low density lipoprotein receptor family member. J Biol Chem 271:24761-24768.

Yan R, Bienkowski MJ, Shuck ME, Miao H, Tory MC, Pauley AM, Brashier JR, Stratman NC, Mathews WR, Buhl AE, Carter DB, Tomasselli AG, Parodi LA, Heinrikson RL, Gurney ME (1999) Membrane-anchored aspartyl protease with Alzheimer's disease $\beta$-secretase activity. Nature 402: 533-537.

Zhu Y, Doray B, Poussu A, Lehto VP, Kornfeld S (2001) Binding of GGA2 to the lysosomal enzyme sorting motif of the mannose 6-phosphate receptor. Science 292:1716-1718. 